\title{
Development of an in vitro Test Procedure to Determine the Direct Infrared A Protection of Sunscreens and Non-Cosmetic Samples
}

\author{
Jan Carlos Quistorf ${ }^{\mathrm{a}} \quad$ Dieter Kockott $^{\mathrm{b}}$ Birgit Garbe ${ }^{\mathrm{a}} \quad$ Ulrike Heinrich $^{\mathrm{a}}$ \\ Hagen Tronnier ${ }^{\mathrm{a}}$ Nicole Braun ${ }^{\mathrm{a}}$ \\ a Institute for Experimental Dermatology, DermaTronnier, Witten/Herdecke University, Witten, and \\ bUV-Technik, Hanau, Germany
}

\section{Keywords}

Infrared A transmittance - Sunscreen and textile infrared A protection $\cdot$ In vitro measurement

\begin{abstract}
Background: Every day human skin is exposed to infrared $A$ (IRA) radiation as part of the natural sun rays. As IRA radiation accounts for around one third of the solar radiation, it has gained great attention concerning its effects on the human body and skin. In the past few years it has been discussed controversially whether IRA radiation (of solar origin) is harmful or not. Nonetheless, there are several sunscreens on the German market that claim IRA protection for themselves. Aims: The present study seeks to find an experimental set-up and a test procedure for the determination and quantification of direct IRA protection (realized via reflection or absorption of the IRA radiation), since to our knowledge these do not yet exist. Methods: In this study we proved the usability of a set-up consisting of a light source, an IRA-transmissible filter system and a sensor unit, for the determination and quantification of the IRA protection of cosmetic and non-cosmetic samples. Results/Conclusion: The applicability of the IRA emission of the light source, the spectral detec-
\end{abstract}

\section{KARGER}

(C) 2017 S. Karger AG, Basel

E-Mail karger@karger.com

www.karger.com/spp tor, transmissivity of the filter systems and the sample carriers could be validated. This experimental set-up can be used as an in vitro test procedure for the determination of direct IRA protection.

(c) 2017 S. Karger AG, Basel

\section{Introduction}

Human skin is exposed to infrared A (IRA) radiation (780-1,400 nm) on a daily basis. This amounts to approximately one third of the global solar radiation [1]. Many studies and examinations were carried out in order to identify and ascertain effects of this radiation on the human body and skin. These studies showed different results, including the IRA-induced generation of reactive oxygen species, a wide range of gene regulations (particularly but not limited to the induction of matrix metalloproteinase-1) and thus a contribution to skin ageing [24]. However, the influence of heat on these findings is being discussed controversially [5-7]. Several studies examined the efficacy of different protective measures against IRA-mediated skin damage $[8,9]$ and recommend IRA protection by using light reflectors or anti-ox- 
Table 1. Classification of the tested sunscreen products with a brief description based on an organoleptic assessment and the SPF as labelled by the manufacturer

\begin{tabular}{ll}
\hline No. & Brief description \\
\hline Sunscreens claiming IRA protection \\
1 & White cream, SPF 30 \\
2 & White cream, SPF 30 \\
3 & White cream, SPF 10 \\
4 & White cream, SPF 30 \\
5 & White cream, SPF 50 \\
6 & White cream, SPF 30 \\
7 & White cream, SPF 30 \\
8 & White emulsion, SPF 30 \\
\hline Sunscreens without claimed IRA protection \\
9 & White cream, SPF 30 \\
10 & White cream, SPF 50 \\
11 & Yellowish liquid, SPF 30 \\
12 & Tinted paste, SPF 50+ \\
13 & Whitish transparent cream, SPF 50 \\
14 & White cream, SPF 20 \\
15 & White cream, SPF 50+ \\
16 & White emulsion, SPF 30 \\
\hline Tinted daily skin care products with SPF \\
17 & Tinted cream, SPF 40 \\
18 & Tinted cream, SPF 35 \\
19 & Lightly tinted cream, SPF 15 \\
20 & Tinted cream, SPF 15 \\
\hline Make-up foundation with SPF \\
21 & Tinted emulsion, SPF 15 \\
\hline & \\
\hline
\end{tabular}

Table 2. Classification of the tested fabrics with a brief description, based on the labelling of the manufacturer

\begin{tabular}{ll}
\hline No. & Brief description \\
\hline New wool & Black, $300 \mathrm{~g} / \mathrm{m}^{2}$ \\
1 & Brown, $380 \mathrm{~g} / \mathrm{m}^{2}$ \\
2 & Brown, $500 \mathrm{~g} / \mathrm{m}^{2}$ \\
3 & Grey, $800 \mathrm{~g} / \mathrm{m}^{2}$ \\
4 & \\
\hline Canvas & Brown, $300 \mathrm{~g} / \mathrm{m}^{2}$ \\
5 & White $340 \mathrm{~g} / \mathrm{m}^{2}$ \\
6 & Grey, $650 \mathrm{~g} / \mathrm{m}^{2}$ \\
7 & \\
\hline Silk & White $68 \mathrm{~g} / \mathrm{m}^{2}$ \\
8 &
\end{tabular}

The fabric weight is given in grams per square metre. The higher the value, the higher the density of the fabric. idants $[4,10,11]$. Moreover there are studies that negate the aforementioned negative effects of IRA radiation on human skin $[6,12,13]$. Some studies even showed a benefit of IRA radiation for human skin, as it may lower the UVB cytotoxicity $[14,15]$. Accordingly, there is a necessity for a definite assessment of the harmfulness of IRA radiation and therewith the need for IRA protection for human skin.

Since several sunscreens on the German market claim IRA protection for themselves, a test method to assess and quantify this IRA protection is needed. This IRA protection can be realized in 2 ways. One way is to use IRA-reflecting or -absorbing materials in order to provide a direct IRA protection (comparable to UV protection). The other way is to use anti-oxidants and radical scavengers in order to compensate the aforementioned negative effects of the IRA radiation on human skin (indirect IRA protection).

The objective of the present study was to develop and validate an experimental set-up to quantify the direct IRA protection of sunscreens and non-cosmetic samples.

This is of great relevance as, to our knowledge, there is no such test procedure yet.

The samples were analysed concerning their transmittance for IRA radiation. For this purpose an experimental set-up consisting of a light source, a filter system, and a sensor unit was used.

\section{Materials and Methods}

\section{Chemicals}

Glycerol, medium-chain triglycerides (Miglyol 812) and lowviscosity paraffin wax were supplied by Caesar \& Loretz (Hilden, Germany), white petroleum jelly by Henry Lamotte Oils (Bremen, Germany), and distilled water by B. Braun (Melsungen, Germany).

Sample Carriers

Polymethylmethacrylate (PMMA) slides with a surface area of $25 \mathrm{~cm}^{2}$, a thickness of $2 \mathrm{~mm}$, and a roughness of $2 \mu \mathrm{m}$ were used for the analysis of the sunscreens in this study. They were purchased from Schönberg (Hamburg, Germany).

\section{Samples}

Of the 21 sunscreens which were chosen for analysis, 8 claimed IRA protection for themselves, 5 were tinted, 1 to a lesser extent. These are described in Table 1.

In addition, 8 textile samples were chosen, 4 of which were made of wool, 3 were made of linen and 1 was made of silk. All 8 samples were of different colours. These are described in Table 2.

\section{Components of the Experimental Set-Up}

The experimental set-up comprised a light source Halospot 70 50-W 12-V $8^{\circ}$ BA15D made by Osram (Munich, Germany), a filter
Quistorf/Kockott/Garbe/Heinrich/ Tronnier/Braun 
system, consisting of a black glass filter RG 780/3 from Schott (Mainz, Germany), and a glass cuvette filled with distilled water (resulting thickness of the water column: $10 \mathrm{~mm}$ ), a sample holder, and a sensor unit. The sensor unit consisted of an integral detector ILT SCS695/W and a photometer ILT1400, both obtained from International Light Technologies (Peabody, MA, USA).

\section{Sample Preparation}

The application amount of the sunscreen samples was $1.00 \mathrm{mg} /$ $\mathrm{cm}^{2}$ with a tolerance of $0.02 \mathrm{mg} / \mathrm{cm}^{2}$ [16]. The application procedure used has been shown to be the most appropriate in preceding studies [17]. It was performed in a way that 25 spots (5 in a row) were applied onto the sample carriers and spread with the forefinger, following a defined and standardized procedure. This procedure included a standardized pressure during application (monitored with a scale) and the time for spreading the sample. At first the sunscreen is spread with small circular movements for $10 \mathrm{~s}$. After that, the sunscreen is spread horizontally and vertically performing 5 sets of each 10 horizontal and 10 vertical spreading movements for $50 \mathrm{~s}$ in total. The vertical and horizontal spreading directions remained consistent throughout the 5 sets. The pressure, measured with a scale, was kept at about $150 \mathrm{~g}$. Directly after the application, the samples were stored in a drying cabinet at $30^{\circ} \mathrm{C}$ for $15 \mathrm{~min}$.

\section{Spectral Measurement}

In order to validate the usability of the single components of the experimental set-up as well as the choice of the blank sample and the sample carriers, the spectral transmittance was measured using a double monochromator spectroradiometer, type Spectro 320 D from Instrument Systems (Munich, Germany).

\section{Measurement}

Every sunscreen sample, prepared as described above, was located in the optical path of the experimental set-up, and the irradiance behind the sample was measured. Immediately after this measurement, a second sample carrier with applied glycerol (as a blank sample) was located in the optical path, and the irradiance behind this blank sample was measured. This blank sample was prepared according to the DIN EN ISO 24443:2012 [18].

The textile samples were fixated in the optical path single-layered and crease-free without a sample carrier. Hence the blank measurement was performed with air as blank sample.

The transmittance of the samples was determined as the quotient of the irradiance behind the sample and the blank sample.

\section{Measurement of Samples}

In a first screening the IRA transmittance of the 21 sunscreens was determined with a sample size of 3 . Subsequently, sunscreens with an IRA transmittance lower than $90 \%$ were chosen and underwent a second cycle of 3 measurements, leading to an overall sample size of 6 .

The textile samples were analysed with a sample size of 3 .

\section{Statistical Analysis}

Descriptive statistics with mean, standard deviation (SD) and relative standard deviation (RSD) is calculated for all measured samples.

The following statistical analysis was performed to characterize the sensitivity of the experimental set-up and not to assess the IRA

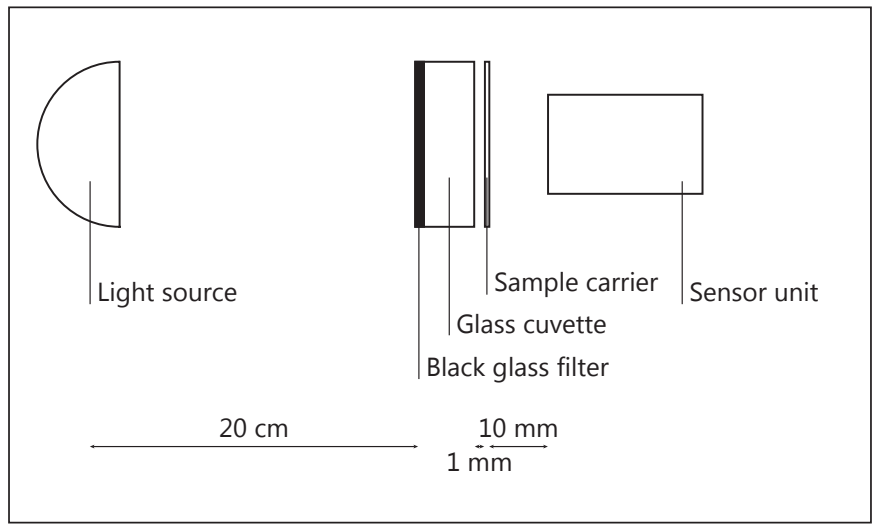

Fig. 1. Schematic representation of the experimental set-up (not true to scale).

protection properties of the sunscreen and textile samples. The variability of the results is assumed to derive from singular and non-systematic application and measurement errors. These are supposed to follow a gaussian distribution.

For this purpose the 1-sided 1-sample $t$ test was used to investigate the null hypothesis that the sample has no IRA-filtering attributes, meaning a transmittance of $100 \%$, against the alternative hypothesis that the sample has IRA-filtering attributes resulting in a transmittance less than $100 \%$. Results were considered statistically significant at the $5 \%$ level $(p<0.05)$.

\section{Results}

\section{Experimental Set-Up}

The experimental set-up, as described above, consisted of the light source Osram Halospot $7050-\mathrm{W} 12-\mathrm{V} 8^{\circ}$ BA15D, which does not need preheating time and emits a broad spectrum of electromagnetic radiation. The filter system consisted of the black glass filter Schott RG 780/3, a 3-mm-thick glass filter for filtering the radiation with wavelengths shorter than $780 \mathrm{~nm}$ and a glass cuvette filled with distilled water. The cuvette had outer dimensions of $55 \times 15 \times 53 \mathrm{~mm}$, leading to a thickness of the water column of $10 \mathrm{~mm}$. This part of the filter system was used to filter the radiation with wavelengths larger than 1,400 $\mathrm{nm}$. The sensor unit contained the photometer ILT1400 linked with the detector ILT-SCS695/W. The detector records the wavelength spectrum from 770 to $3,000 \mathrm{~nm}$ in the range of $0.0857-414 \mathrm{~mW} / \mathrm{cm}^{2}$.

The components were arranged as shown in Figure 1. The distances between the single components of the experimental set-up were maintained identical throughout all measurements and as follows: the distance between the light source and the filter system was $20 \mathrm{~cm}$. The black 
Fig. 2. The spectral irradiance with no sample (air), a roughened sample carrier (PMMA rough), a smooth PMMA slide (PMMA smooth), a roughened sample carrier with glycerol (rough + glyc.) and a smooth PMMA slide with glycerol (smooth + glyc.) as a function of the wavelength.
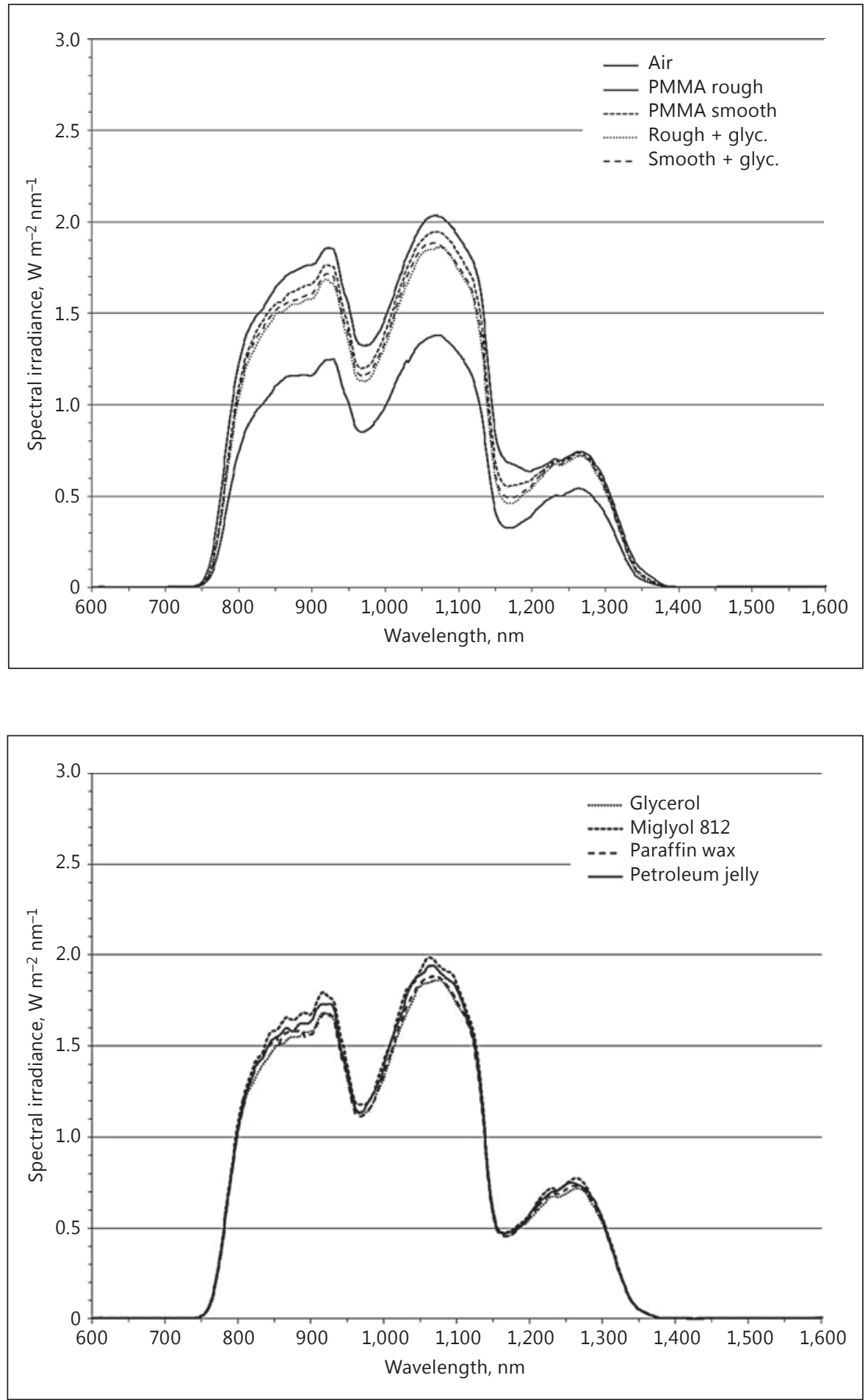

Fig. 3. The spectral irradiance with glycerol, medium-chained triglycerides (Miglyol 812 ), low-viscosity paraffin wax and white petroleum jelly as a function of the wavelength.

\section{Spectral Measurement}

The transmissivity of the filter system and the sample carriers for IRA radiation was proven in this spectral measurement. 
Fig. 4. The spectral irradiance using waterfilled cuvettes with a thickness of the resulting water column of 10 and $20 \mathrm{~mm}$ as a function of the wavelength.

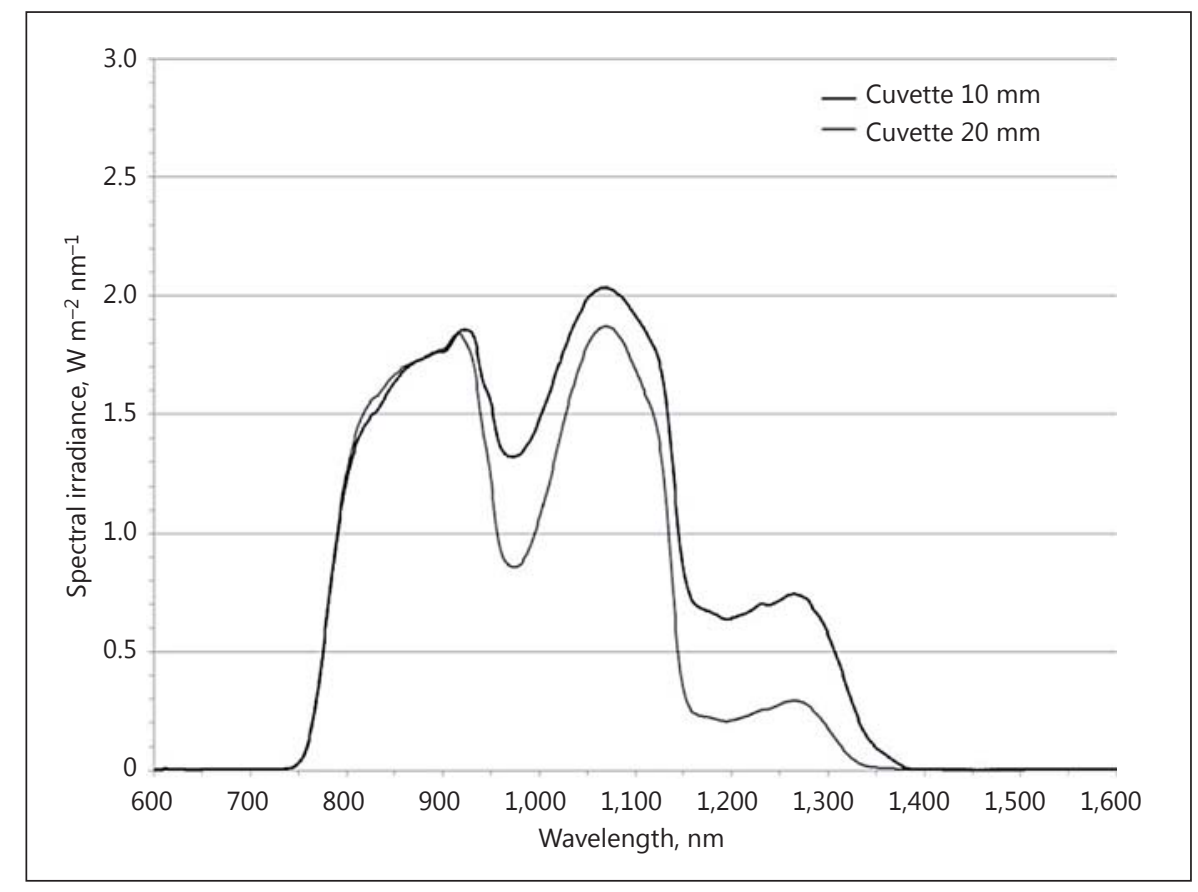

First of all, the IRA-transmitting qualities of the filter system and the sample carriers as well as the IRA emission of the light source were examined (Fig. 2).

The sample carrier (PMMA rough) clearly showed a weaker transmissivity compared to air, presumably due to the roughened surface. However, it was possible to nearly equalize this effect by applying one of the blank samples. The resulting transmittance of the roughened sample carrier with glycerol (rough + glyc.) was almost the same as the transmittance of the smooth PMMA slides (PMMA smooth) or smooth PMMA slides with glycerol applied to it (smooth + glyc.).

As can be seen in Figure 2, the filter system is able to filter radiation with wavelengths shorter than about $780 \mathrm{~nm}$ and larger than about 1,400 $\mathrm{nm}$.

The light source showed a broad emission of radiation throughout the whole IRA wavelength range.

The 4 blank samples showed virtually the same transmissivity (Fig. 3), which is why glycerol, that is well known and used in the in vitro evaluation of ultraviolet filters, was chosen for use in this study.

The use of cuvettes with different light path lengths was examined (Fig. 4). The cuvette with a light path of $20 \mathrm{~mm}$ showed larger filtering attributes in the range of $1,350-1,400 \mathrm{~nm}$, which is part of the desired IRA spectrum. This is why the cuvette with a light path of $10 \mathrm{~mm}$ was chosen for this study.

Development of IRA Protection Testing in vitro
The light source was examined concerning a shift of the spectral irradiance when using different irradiation intensities (maximum and minimum). As can be seen in Figure 5, no relevant shift of the spectral irradiance (except for the differences in the absolute values, which correspond to the expectation) was observed.

These spectral measurements proved the applicability of all components of the experimental set-up for the determination of a sample's IRA transmittance. This was necessary in order to prove the adequacy of using an integral detector instead of a spectral detector for future measurements.

\section{Determination of the IRA Transmittance}

The determination of the IRA transmittance of the 21 sunscreen samples and the 8 textile samples was carried out in order to investigate the applicability of the experimental set-up and especially the reproducibility of the obtained results. Furthermore, it was to be investigated whether the set-up in the present study is applicable for cosmetic as well as non-cosmetic samples.

\section{Sunscreen Samples}

The screening of the sunscreen products led to the following results (Table 3). The sunscreens claiming protection against IRA showed a mean IRA transmittance between 95 and $98 \%$ and an RSD between 0.1 and $0.9 \%$.

Skin Pharmacol Physiol 2017;30:171-179 DOI: $10.1159 / 000475474$ 
Fig. 5. The spectral irradiance using maximum and minimum irradiance of the light source as a function of the wavelength.

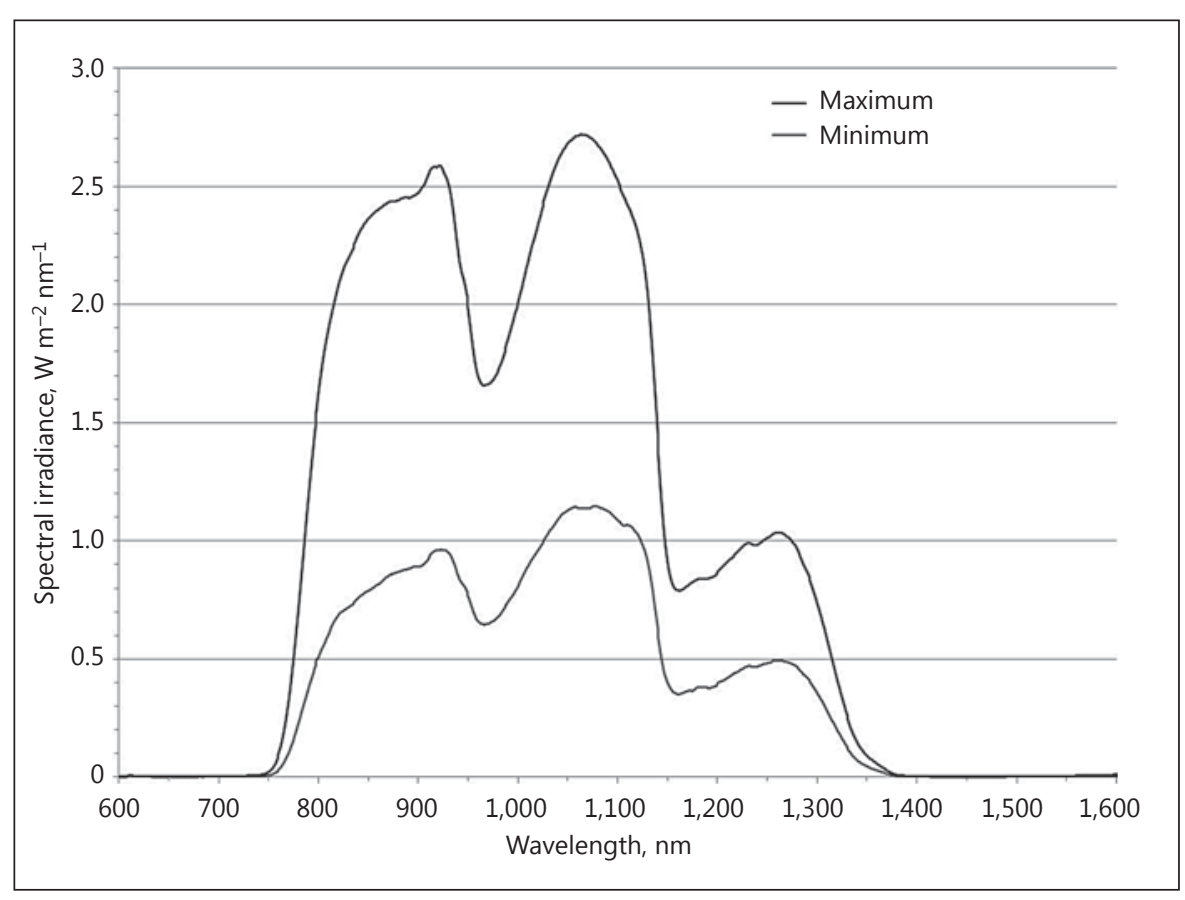

Table 3. IRA transmittance of sunscreen products

\begin{tabular}{|c|c|c|c|c|}
\hline Product class & Mean, \% & SD & RSD, \% & $p$ value \\
\hline \multicolumn{5}{|c|}{ Sunscreens claiming IRA protection } \\
\hline 1 & 97.6 & 0.7 & 0.7 & $<0.05$ \\
\hline 2 & 98.2 & 0.4 & 0.4 & $<0.01$ \\
\hline 3 & 95.6 & 0.7 & 0.7 & $<0.01$ \\
\hline 4 & 94.4 & 0.1 & 0.1 & $<0.01$ \\
\hline 5 & 95.8 & 0.6 & 0.7 & $<0.01$ \\
\hline 6 & 94.8 & 0.8 & 0.9 & $<0.01$ \\
\hline 7 & 96.5 & 0.2 & 0.2 & $<0.01$ \\
\hline 8 & 95.1 & 0.6 & 0.6 & $<0.01$ \\
\hline \multicolumn{5}{|c|}{ Sunscreens without claimed IRA protection } \\
\hline 9 & 91.6 & 2.3 & 2.5 & $<0.05$ \\
\hline 10 & 97.3 & 0.5 & 0.5 & $<0.01$ \\
\hline 11 & 100.0 & 1.6 & 1.6 & ns \\
\hline 12 & 49.1 & 1.7 & 3.4 & $<0.01$ \\
\hline 13 & 99.3 & 1.7 & 1.7 & ns \\
\hline 14 & 94.8 & 1.9 & 2.0 & $<0.05$ \\
\hline 15 & 95.1 & 0.5 & 0.5 & $<0.01$ \\
\hline 16 & 99.1 & 1.2 & 1.2 & ns \\
\hline \multicolumn{5}{|c|}{ Tinted daily skin care products with SPF } \\
\hline 17 & 73.8 & 1.9 & 2.6 & $<0.01$ \\
\hline 18 & 88.3 & 0.3 & 0.4 & $<0.01$ \\
\hline 19 & 96.7 & 1.7 & 1.8 & $<0.05$ \\
\hline 20 & 87.0 & 2.7 & 3.1 & $<0.01$ \\
\hline \multicolumn{5}{|c|}{ Makeup foundation with SPF } \\
\hline 21 & 66.7 & 0.9 & 1.4 & $<0.01$ \\
\hline
\end{tabular}

Shown is the descriptive statistics with $p$ values $(n=3)$.
Skin Pharmacol Physiol 2017;30:171-179 DOI: $10.1159 / 000475474$
Seven out of the 8 sunscreens without claimed IRA protection showed a mean IRA transmittance between 92 and $100 \%(0.5-2.5 \%$ RSD). However, the mean IRA transmittance of 1 sunscreen without claimed IRA protection lay by $49 \pm 3.4 \%$ RSD. That is, however, a tinted paste.

The tinted daily skin care products with SPF showed a mean IRA transmittance between 74 and 97\% (0.4-3.1\% RSD).

The mean IRA transmittance of the make-up foundation with SPF lay at $67 \pm 0.9 \%$ RSD.

The results from 18 out of 21 tested samples differed statistically significantly from a $100 \%$ transmittance (Table 4; Fig. 6).

In order to verify the results of the sunscreen samples with an IRA transmittance lower than $90 \%$, a second cycle of 3 measurements was performed. Here, the retested products are 1 tinted sunscreen paste without claimed IRA protection, 3 tinted daily skin care products with SPF and the make-up foundation with SPF.

The previously obtained results could be verified by the further measurements (Table 5; Fig. 7).

\section{Textile Samples}

The textile samples examined were new wool, canvas and silk. Here, the density of the new wool tested ranged from 300 to $800 \mathrm{~g} / \mathrm{m}^{2}$ : the colours were black, grey, and 
Fig. 6. IRA transmittance of the sunscreens products. Columns represent the average results of 3 samples. The 5 sunscreens with a transmittance below 90\% (dotted line) are diagonally striped. $* p<0.05$, $* * p<0.01$ : statistical significance (1-sided, 1 -sample $t$ test); ns, non-significant.

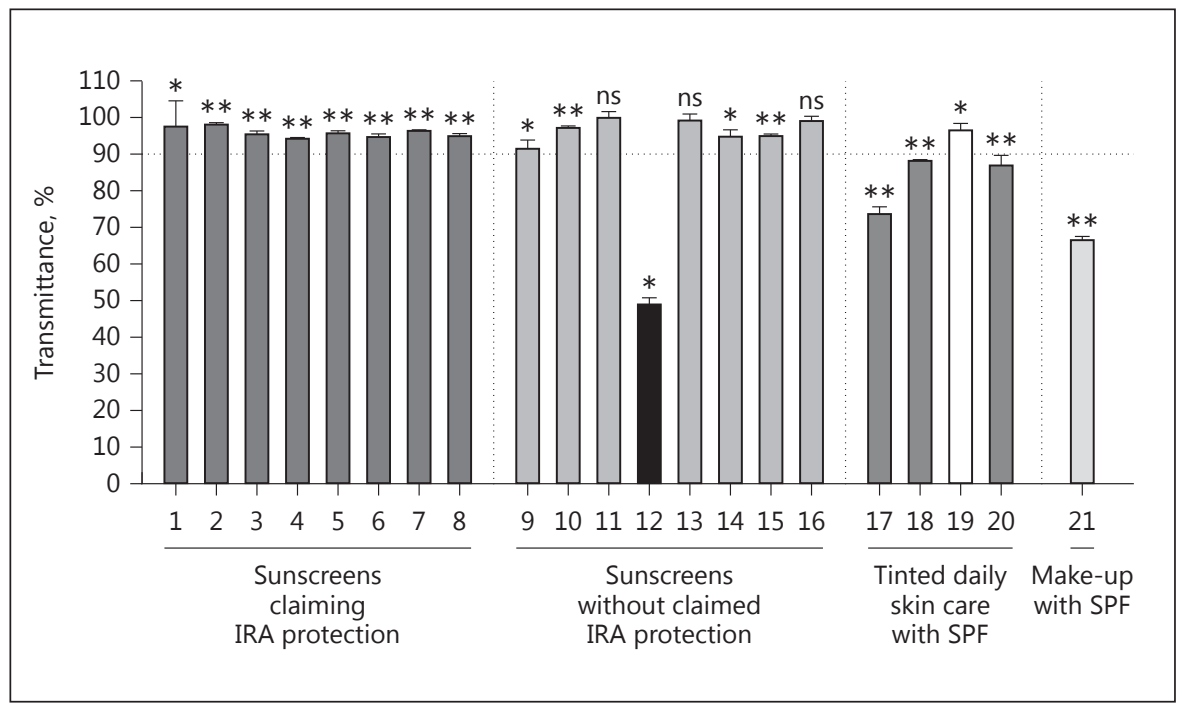

brown. The mean IRA transmittance of the new wool in the range of $300-500 \mathrm{~g} / \mathrm{m}^{2}$ was $16-26 \%$ (0.4-4.0\% RSD). However, the pure wool with a density of $800 \mathrm{~g} / \mathrm{m}^{2}$ showed a mean IRA transmittance of $7 \pm 8.5 \% \mathrm{RSD}$. The density of the canvas tested ranged from 300 to $650 \mathrm{~g} / \mathrm{m}^{2}$, and the colours were grey, brown, and white. The canvas sample with a density from 300 to $340 \mathrm{~g} / \mathrm{m}^{2}$ showed a mean IRA transmittance between 25 and 27\% (1.9-3.1\% RSD). However, the canvas with a density of $650 \mathrm{~g} / \mathrm{m}^{2}$ showed a mean IRA transmittance of $5 \pm 6.2 \% \mathrm{RSD}$. In addition, a silk sample was tested. The IRA transmittance of the white silk with a density of $68 \mathrm{~g} / \mathrm{m}^{2}$ lay at $35 \pm 4.4 \%$ RSD. The results from all tested fabric samples differed statistically significantly from a $100 \%$ transmittance with $p$ values $>0.001$ (Table 5; Fig. 8).

In general, it is noticeable that the density of the fabrics correlates with the IRA transmittance.

\section{Discussion}

The objective of this study was to develop and validate an in vitro test procedure in order to quantify the direct IRA protection of sunscreens and non-cosmetic samples.

A few study groups introduced different experimental set-ups and test procedures for the determination and characterization of the indirect IRA protection, e.g., the determination of a radical protection factor and skin radical formation in the IR range [8]. In 2014 Dueva-Koganov et al. [19] proposed a test procedure to ex-

Development of IRA Protection Testing in vitro
Table 4. Sunscreen products with IRA transmittance below $90 \%$

\begin{tabular}{|c|c|c|c|c|}
\hline Product class & Mean, \% & SD & RSD, \% & $p$ value \\
\hline \multicolumn{5}{|c|}{ Sunscreen without claimed IRA protection } \\
\hline 12 & 49.8 & 2.5 & 5.0 & $<0.01$ \\
\hline \multicolumn{5}{|c|}{ Tinted daily skin care products with SPF } \\
\hline 17 & 73.4 & 1.6 & 2.2 & $<0.01$ \\
\hline 18 & 88.3 & 1.1 & 1.3 & $<0.01$ \\
\hline 20 & 87.0 & 1.7 & 2.0 & $<0.01$ \\
\hline \multicolumn{5}{|c|}{ Make-up foundation with SPF } \\
\hline 21 & 66.8 & 0.9 & 1.3 & $<0.01$ \\
\hline
\end{tabular}

Shown is the descriptive statistics with $p$ values $(n=6)$.

Table 5. IRA transmittance of fabrics

\begin{tabular}{lccll}
\hline Product class & Mean, $\%$ & SD & RSD, $\%$ & $p$ value \\
\hline New wool & & & & \\
1 & 18.0 & 0.7 & 4.0 & $<0.01$ \\
2 & 26.1 & 0.1 & 0.4 & $<0.01$ \\
3 & 15.7 & 0.4 & 2.6 & $<0.01$ \\
4 & 7.1 & 0.6 & 8.5 & $<0.01$ \\
\hline Canvas & & & & \\
5 & 24.6 & 0.5 & 1.9 & $<0.01$ \\
6 & 27.4 & 0.8 & 3.1 & $<0.01$ \\
7 & 5.3 & 0.3 & 6.2 & $<0.01$ \\
\hline Silk & & & & \\
8 & 35.2 & 1.6 & 4.4 & $<0.01$ \\
\hline
\end{tabular}

Shown is the descriptive statistics with $p$ values $(n=3)$.

Skin Pharmacol Physiol 2017;30:171-179 


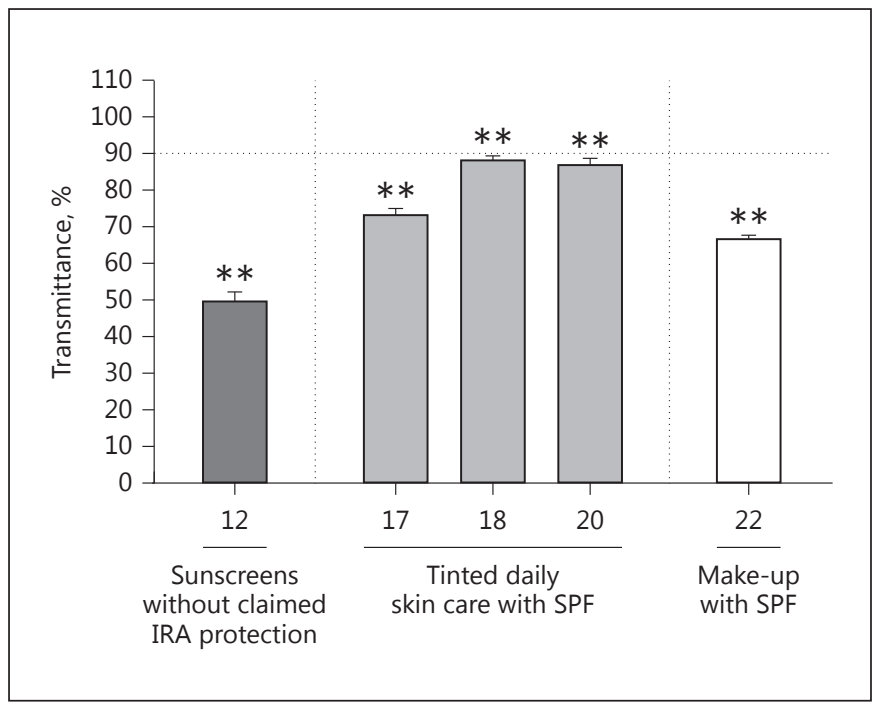

Fig. 7. IRA transmittance of the sunscreen products with a transmittance below 90\%. Columns represent the average results of 6 samples. ${ }^{* *} p<0.01$ : statistical significance (1-sided, 1 -sample $t$ test).

amine sunscreens with regard to their protection against the whole sun spectrum in general and the visible and infrared radiation in particular. In this procedure the radiation is directed through a mirror arrangement and leads through a sample carrier. The radiation is detected with a spectral detector [19]. The advantage of the set-up in the present study is the use of an integral detector in lieu of a spectral detector. This makes the test procedure considerably easier, faster and more cost-efficient.

The applicability of all components was shown and therewith the adequacy of using the above-mentioned integral detector.

In a next step different sunscreen samples and noncosmetic samples were tested with the experimental set-up.

Due to the fact that the deviation from a $100 \%$ transmittance was, in most cases, on a statistically significant level, it can be stated that this experimental set-up can be used in the in vitro determination of the direct IRA protection of sunscreens and non-cosmetic samples.

Almost all sunscreen samples tested, whether with or without claimed IRA protection, showed no direct IRA protection. In general, the transmittance of IRA radiation was more than $90 \%$. Only 3 measured products showed a lower IRA transmittance with values ranging from 49 to $74 \%$. All 3 products were tinted. However, to

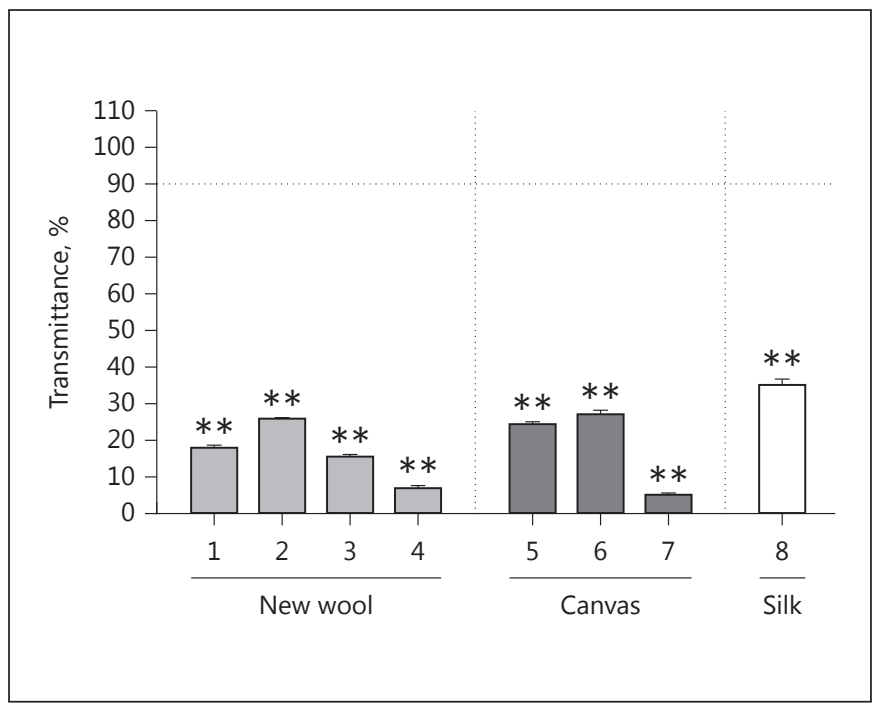

Fig. 8. IRA transmittance of the fabrics. Columns represent the average results of 3 samples. ${ }^{* *} p<0.01$ : statistical significance (1-sided, 1-sample $t$ test).

what extent a reduction in IRA transmission has a biologically meaningful effect on the skin needs further investigation.

The non-cosmetic fabric samples showed noticeably lower transmittances than the sunscreens with IRA transmittance between 5 and $35 \%$. An analysis of the results shows that the dye used has probably a minor effect on the IRA transmittance in contrast to the type of fabric and its thickness.

\section{Conclusion}

Despite the fact that we still lack a definite assessment of the harmfulness of IRA radiation on human skin, we managed to provide and validate an in vitro test procedure for the determination of direct IRA protection. As long as we have sunscreens on the market that claim IRA-protective attributes, there is a need for a valid test procedure for quantification.

Furthermore, we showed that this test procedure is also applicable for non-cosmetic samples. Irrespective of potential future findings concerning the harmfulness of IRA radiation for human skin, this can therefore be used for the determination of IRA-filtering attributes, e.g., of window foils for greenhouses etc. 


\section{Acknowledgement}

The authors would like to thank Dr. Helmut Piazena for performing the spectral measurements in this study and Dipl.-Stat. Carmen Theek for her assistance concerning the statistical evaluation of the results.

\section{Statement of Ethics}

No ethical conflicts exist, because only in vitro tests with sunscreens and canvas were performed.

\section{Disclosure Statement}

The authors declare that no conflicts of interest exist.

\section{References}

1 Schroeder P, Pohl C, Calles C, Marks C, Wild $S$, Krutmann J: Cellular response to infrared radiation involves retrograde mitochondrial signaling. Free Radic Biol Med 2007;43:128135.

2 Calles C, Schneider M, Macaluso F, Benesova T, Krutmann J, Schroeder P: Infrared A radiation influences the skin fibroblast transcriptome: mechanisms and consequences. J Invest Dermatol 2010;130:1524-1536.

3 Schroeder P, Lademann J, Darvin ME, Stege H, Marks C, Bruhnke S, Krutmann J: Infrared radiation-induced matrix metalloproteinase in human skin: implications for protection. J Invest Dermatol 2008;128:2491-2497.

4 Robert C, Bonnet M, Marques S, Numa M, Doucet O: Low to moderate doses of infrared A irradiation impair extracellular matrix homeostasis of the skin and contribute to skin photodamage. Skin Pharmacol Physiol 2015; 28:196-204.

5 Zastrow L, Groth N, Klein F, Kockott D, Lademann J, Renneberg R, Ferrero L: The missing link - light-induced (280-1,600 nm) free radical formation in human skin. Skin Pharmacol Physiol 2009;22:31-44.

6 Piazena H, Pittermann W, Muller W, Jung K, Kelleher DK, Herrling T, Meffert P, Uebelhack R, Kietzmann M: Effects of water-filtered infrared-A and of heat on cell death, inflammation, antioxidative potential and of free radical formation in viable skin - first results. J Photochem Photobiol B 2014;138: 347-354.
7 Jung T, Hohn A, Piazena H, Grune T: Effects of water-filtered infrared A irradiation on human fibroblasts. Free Radic Biol Med 2010;48: 153-160.

8 Meinke MC, Syring F, Schanzer S, Haag SF, Graf R, Loch M, Gersonde I, Groth N, Pflucker F, Lademann J: Radical protection by differently composed creams in the UV/VIS and IR spectral ranges. Photochem Photobiol 2013;89:1079-1084.

9 Grether-Beck S, Marini A, Jaenicke T, Krutmann J: Photoprotection of human skin beyond ultraviolet radiation. Photodermatol Photoimmunol Photomed 2014;30:167-174.

10 Grether-Beck S, Marini A, Jaenicke T, Krutmann J: Effective photoprotection of human skin against infrared A radiation by topically applied antioxidants: results from a vehicle controlled, double-blind, randomized study. Photochem Photobiol 2015;91:248-250.

11 Schroeder P, Krutmann J: What is needed for a sunscreen to provide complete protection. Skin Ther Lett 2010;15:4-5.

12 Gebbers N, Hirt-Burri N, Scaletta C, Hoffmann G, Applegate LA: Water-filtered infrared-A radiation (WIRA) is not implicated in cellular degeneration of human skin. Ger Med Sci 2007;5:Doc08.
13 Piazena H, Kelleher DK: Effects of infrared-A irradiation on skin: discrepancies in published data highlight the need for an exact consideration of physical and photobiological laws and appropriate experimental settings. Photochem Photobiol 2010;86:687-705.

14 Frank S, Menezes S, Lebreton-De Coster C, Oster M, Dubertret L, Coulomb B: Infrared radiation induces the $\mathrm{p} 53$ signaling pathway: role in infrared prevention of ultraviolet $\mathrm{B}$ toxicity. Exp Dermatol 2006;15:130-137.

15 Hohn A, Hartmann P, Gebhart V, Sonntag J, Grune T, Jung T: Actual isothermal effects of water-filtered infrared A irradiation. Photochem Photobiol 2015;91:887-894.

16 Heinrich U, Tronnier H, Kockott D, Kuckuk $\mathrm{R}$, Heise HM: Comparison of sun protection factors determined by an in vivo and different in vitro methodologies: a study with 58 different commercially available sunscreen products. Int J Cosmet Sci 2004;26:79-89.

17 Reckmann V: Photostabilität von Lichtschutzmitteln - Bestimmung und Bewertung; $\mathrm{PhD}$ thesis, Institute of Experimental Dermatology, University of Witten/Herdecke, 2016.

18 Din en Iso 24443: 2012 determination of sunscreen UVA photoprotetion in vitro. Berlin, 2013.

19 Dueva-Koganov O, Duev A, Turner R, Micceri S: In vitro evaluation of potential protection provided by topical products against full solar and visible plus infrared radiation. Household Pers Care Today 2014;9:27-43. 